\title{
Realization of spectrally engineered semiconductor Fabry-Perot lasers with narrow geometrical tolerances
}

\author{
Göran Adolfsson, ${ }^{1, a)}$ Jörgen Bengtsson, ${ }^{1}$ Åsa Haglund, ${ }^{1}$ Bengt Nilsson, ${ }^{2}$ \\ and Anders Larsson ${ }^{1}$ \\ ${ }^{1}$ Photonics Laboratory, Department of Microtechnology and Nanoscience-MC2, Chalmers University of \\ Technology, SE-41296 Göteborg, Sweden \\ ${ }^{2}$ Nanofabrication Laboratory, Department of Microtechnology and Nanoscience-MC2, Chalmers University of \\ Technology, SE-41296 Göteborg, Sweden
}

(Received 17 March 2011; accepted 6 April 2011; published online 10 May 2011)

\begin{abstract}
Spectrally engineered semiconductor Fabry-Perot laser resonators are designed to enhance the optical feedback for selected longitudinal modes, which thereby require less gain for lasing. This is achieved by introducing refractive index perturbations along the length of the resonator. However, the physical realization of these resonators is a challenge because of very narrow tolerances; in particular the need for precise positioning of the end facets of the resonator in relation to the perturbations, and the excess propagation loss associated with the perturbations, has been a major concern. We report on a method to achieve high-quality end facet mirrors enabling precise positioning relative to the perturbations, the latter which are realized as lateral corrugations of the waveguide. Measurements show that the mirror quality is comparable to that of cleaved mirrors and that the additional loss introduced by the perturbations adds $\lesssim 10 \mathrm{~cm}^{-1}$ to the overall propagation loss, provided that the perturbations are densely enough spaced along the resonator. This implies that the number of perturbations should be large, which is beneficial for the realization of strongly perturbed resonators enabling the most flexible engineering of the spectral properties of the laser. (C) 2011 American Institute of Physics. [doi:10.1063/1.3587359]
\end{abstract}

\section{INTRODUCTION}

The longitudinal modes in a conventional Fabry-Perot laser (FPL) are defined by the mirror separation $L$, with resonance wavelengths given by $\lambda_{m}=2 n L / m$, where $\lambda_{m}$ is the wavelength of the $m^{\text {th }}$ mode, $m=1,2, \ldots$, and $n$ is the effective mode index inside the FP resonator. The only feedback mechanism in an FPL is provided by the reflections at the end facets. Since this feedback is equally strong for all wavelengths the threshold gain needed for lasing is the same for all longitudinal modes. In this work, we use the term threshold gain spectrum for the (net modal) threshold gain of the various longitudinal modes in some wavelength region of interest (coinciding with the gain bandwidth of the active medium). For the FPL, the threshold gain spectrum is thus defined at the resonance wavelengths, with identical values for the threshold gain for all modes. The only wavelength selectivity in an FPL is thus provided by the gain medium and FPLs therefore emit in multiple longitudinal modes since the mode spacing is much narrower than the gain bandwidth. By introducing perturbations of the mode index along the length of the resonator an extra feedback mechanism is employed; the optical field will be partially reflected at each interface between segments with an unperturbed and perturbed mode index. The extra feedback can be used to tailor the threshold gain of the longitudinal modes, in order to create an almost arbitrary threshold gain spectrum. Such a spectrally engineered

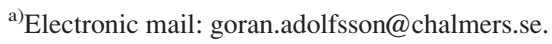

Fabry-Perot resonator (SE-FPR) can, e.g., be designed to preferentially lower the threshold gain for one or several longitudinal modes. In this way, ridge waveguide lasers with strong single mode operation as well as those with two-color operation, i.e., simultaneous emission of two wavelengths, have been realized. ${ }^{1-3}$ These lasers were conventional (FabryPerot) ridge waveguide lasers where the spectral properties were engineered by introducing short trenches, or perturbations, at a few (normally less than 50) positions along the ridge, in the upper part of the ridge. The trench changes the cross section of the waveguide and thus the effective index is different in the trenched region. Previously, the design methods required that the perturbation was not too strong-multiple reflections between perturbations were ignored. This implies that the number of perturbations is not too large. Recently, though, a design method for arbitrarily strongly perturbed waveguides, accounting for any number of multiple reflections, was developed. ${ }^{4}$ In this treatment, the perturbed and unperturbed waveguide sections were viewed as cascaded subcavities with different effective indices. The intricate coupling between all the subcavities was accounted for using a transfer matrix formalism. Strongly perturbed resonators allow for an almost arbitrary threshold gain spectrum to be created and can use up to $\sim 1000$ perturbations. It should also be noted, that even in the strongly coupled case the end facets play an important role-unlike conventional distributed Bragg reflector mirror resonators where the optical field falls off toward the end facets, in an SE-FPR there can be a significant optical field incident on the end facets. Therefore, the position 
and quality of the end facets are critical for this type of resonator. In the realization of lasers with SE-FPRs, there are three critical steps:

A) The numerical design of the size and location of the perturbed parts of the waveguide;

B) The physical realization of the perturbed resonator geometry, including the end facets;

C) Control of the material properties determining the optical gain and refractive index (dispersion).

Step A is a purely numerical procedure, and was described in Ref. 4 for a strongly perturbed resonator. The waveguide is then mathematically divided into a large number of short segments of equal length, and the design method iteratively determines the optimal effective index of each segment to reach the desired spectral behavior as closely as possible. The lengths of these fundamental "building block" segments are an integer number, $s$, of (approximately) quarter wavelengths in the waveguide. Using a large $s$ in the design means longer and thus fewer subcavities, giving a more weakly perturbed resonator. To simplify the design we consider a binary perturbation, i.e., the effective index of the wave propagating in the segment can be either $n$ ("unperturbed") or $n+\Delta n$ ("perturbed"). In this step, some of the material parameters (which need to be precisely known or controlled) are fed into the design algorithm, most notably the effective refractive index $n$. Step B is the topic of this paper. In this step, one must first determine how the physical perturbation should be realized. As will be shown, in our case we chose it to be a sidewall etch of the ridge of the waveguide, making it narrower, so that the effective index in a perturbed segment is reduced, i.e., $\Delta n<0$. A top view of one such perturbed ridge waveguide is schematically shown in Fig. 1; as can be seen the perturbed segments are so many that they often appear immediately next to each other and thus form longer segments of a narrow ridge. In this case, the unperturbed ridge width was $6 \mu \mathrm{m}$, whereas the perturbed was $3 \mu \mathrm{m}$. Second, processing techniques must be found that enable the realization of these complex structures. Third, a method must be found to lithographically define and subsequently etch the end facet mirrors. This point is critical, since cleaved mirrors can only be positioned with one or a few micrometer accuracy with respect to the perturbations. By far, this is not accurate enough, and therefore cleaved mirrors cannot be used. Figure 2 illustrates the high precision required in the positioning. This example is for a two-color resonator, and the figure shows the error function $E$, which is the rms deviation of the actual threshold gain spectrum from the desired, normalized to its lowest value, i.e., for perfect positioning. The insets also show more directly how the threshold gain spectrum deteriorates as the distance from the end mirror to the nearest perturbation deviates from the design value. As can be seen, for the worst position, one quarter wavelength away from its optimum, the function of the feedback is even reversed-the threshold gain for the two "lasing" modes is now higher than for the other modes. As could be expected there are multiple positions, one half wavelength apart, that would work, but the chance of randomly position both end mirrors at such positions is very

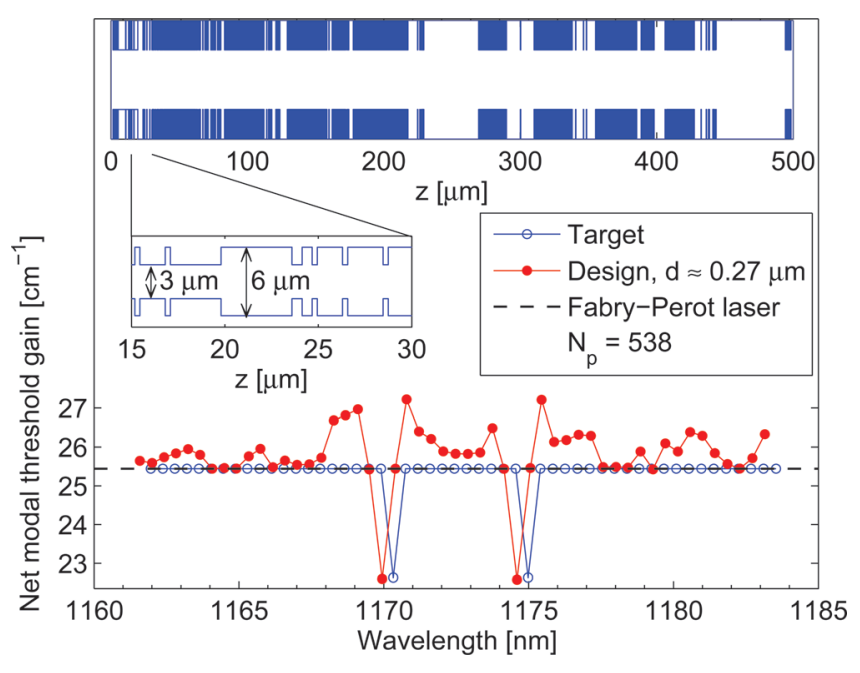

FIG. 1. (Color online) Example of results from the design of an SE-FPR. At the top is shown a schematic top view of the ridge waveguide, with narrow (perturbed) and broad (unperturbed) sections. Below is shown the threshold gain spectrum for the longitudinal modes, obtained in the design. This accurately matches the target, since the target is any threshold value equal to or higher than the indicated value, except for the two wavelengths with a lowered threshold, where the indicated value should be precisely obtained. The slight blueshift of the modes in the design, compared to the target, is caused by the introduction of perturbed segments, with a lower effective index. In the design specification, $s=3$, so that the smallest possible perturbed section had a length $d=270 \mathrm{~nm}$; the longest had a length $20 d=5.4 \mu \mathrm{m}$. In the design $n=3.25$ and $\Delta n=-0.0033$ were used. The total number of perturbed segments in the design solution was $N_{p}=538$.

small; such a strategy for device fabrication would result in an extremely low yield for this reason alone. In addition to the precise positioning, the etched facets must have a smooth surface and a deviation from perfect vertical orientation of less than a few degrees to provide feedback comparable to that of a crystal-oriented cleaved facet. ${ }^{5}$

In this work, we present a fabrication method that involves the use of a single self-aligned metallic mask for definition of both the laterally corrugated, perturbed, ridge waveguide and the etched facets. This offers a precise positioning of the end facets with respect to the perturbations.

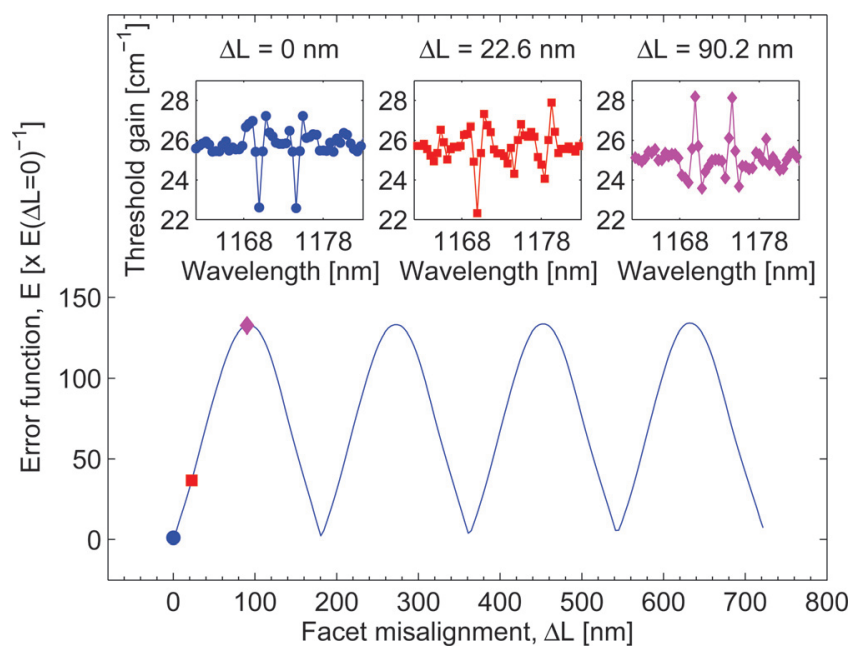

FIG. 2. (Color online) The error $E$ as a function of the error in longitudinal positioning of the facet mirrors relative to the perturbations. The insets show the calculated threshold gain spectra for the longitudinal modes for three different values of positioning error $\Delta L$. 
With the developed fabrication method we achieved highquality etched facets. Moreover, we find, perhaps surprisingly, that the excess propagation loss introduced by the perturbations decreases with the number of perturbations, adding incentive to consider the realization of strongly perturbed laser resonators.

\section{PHYSICAL REALIZATION OF INDEX PERTURBATIONS}

The perturbed resonator segments can be achieved by decreasing the cross section area of the ridge waveguide, either by making it shallower, with a top etch, or narrower, with an etch of the side walls. Top etched perturbations have been successfully implemented in cases where the perturbations have been sparsely positioned, i.e., for weakly perturbed resonators. ${ }^{1-3}$ However, with a sidewall etch it is easier to reach higher spatial resolution, ${ }^{3}$ which is thus preferred for strongly perturbed resonators with their densely positioned perturbations. One example of a more conventional laser resonator using etched sidewall gratings was a distributed feedback laser using a third order grating with a period of $\sim 440 \mathrm{~nm} .{ }^{6}$ As a further complication of the top etch geometry, it may aggravate the current injection through the top contact on the ridge if the perturbations are numerous and densely spaced. For these reasons, we chose to use the sidewall etch as a means to reduce the effective index in the perturbed sections. To calculate the effective indices of the unperturbed ridge, $6 \mu \mathrm{m}$ wide, and the perturbed ridge, $3 \mu \mathrm{m}$ wide, we used the numerical technique described in Ref. 7 to obtain the transverse field profiles of the waveguide modes and their effective indices for different wavelengths. The calculated dispersion curves are shown in Fig. 3, together with an example of an obtained transverse mode profile of the fundamental mode. The epitaxial structure used in the calculations consisted of an InGaAs quantum well active region embedded between AlGaAs cladding layers. Details of the

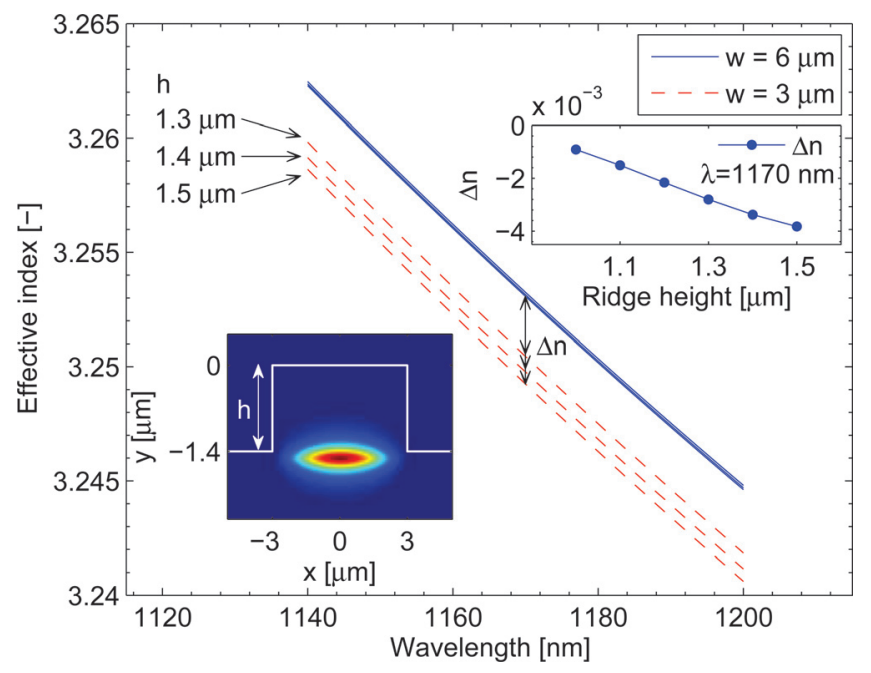

FIG. 3. (Color online) Calculated effective index as a function of wavelength for the fundamental mode in the unperturbed (width $w=6 \mu \mathrm{m}$ ) and perturbed $(w=3 \mu \mathrm{m})$ waveguide sections. The index of the unperturbed waveguide is almost independent of ridge height $h$, unlike that of the perturbed waveguide, as indicated. structure are given in Sec. IV. In the calculations, the material (bulk) dispersion for AlGaAs was taken from Ref. 8. As can be seen in the figure, $\Delta n$ is very nearly constant throughout the considered wavelength region, suggesting that dispersion can be safely neglected for $\Delta n$, and only be considered for the unperturbed effective index $n$. The figure also shows that in order to obtain a large $\Delta n$ the ridge height should be as large as possible, i.e., the ridge should be etched down to just above the active region.

\section{PHYSICAL REALIZATION OF LATERALLY PERTURBED RIDGE WAVEGUIDES AND HIGH-QUALITY END FACET MIRRORS}

It has been shown that facets of good quality can be fabricated using a number of highly anisotropic dry etching techniques in combination with a suitable etch mask. The quality of the dry-etched facet strongly depends on the properties of the etch mask. ${ }^{9-14}$ Corrugations of the mask edges, caused either by mask erosion during etching or originating from the fabrication of the mask itself, are transferred to the facet during the etching process, which may result in both surface roughness and nonverticality of the end facet. Additional roughness may be caused by the post etching processing steps, suggesting that the facet etching should be one of the final steps in the device fabrication. In addition, as mentioned, the etched facet must be precisely positioned with respect to the perturbations. This we solved with a selfaligned process in which a single etch mask was used for dry etching both the laterally corrugated ridge waveguide and the resonator facets.

A schematic drawing of the etch mask is shown in lefthand part of Fig. 4. The main part of it defines the widthmodulated ridge of the waveguide, but near both ends of the resonator, where the facets will be etched, its width is strongly extended, thus defining a waveguide with T-bar shaped ends. The width of the extended waveguide is taken to be much larger than the lateral extension of the optical mode in the waveguide, making sure that the optical field is practically zero on the perimeter of the etched facet mirror. It may be noted that a similar approach has previously been used to fabricate dry etched facets with low surface roughness. ${ }^{10}$ The introduction of the wide T-bar changes slightly the effective index in the waveguide at its very ends, but it can be accounted for in the design. The "length" of the T-bar (i.e., its extension in the longitudinal direction of the waveguide, which should be much shorter than its width) should be just large enough to allow for convenient alignment of the etch masks used for protecting the area near the facet during the waveguide etching, and for protecting the waveguide during the facet etching, as will be described in the next section. In Ref. 10, it was demonstrated that lengths of up to 4 $\mu \mathrm{m}$ would be sufficient to achieve a flat facet, without causing any significant influence on the guided mode. This made it possible for us to use conventional photolithography and mask aligners to align the resist masks along the symmetry line of the T-bar in the extended direction. In order to obtain a well defined etch mask it is desirable to use a mask that is as thin as possible and defined on a planar surface. Many 


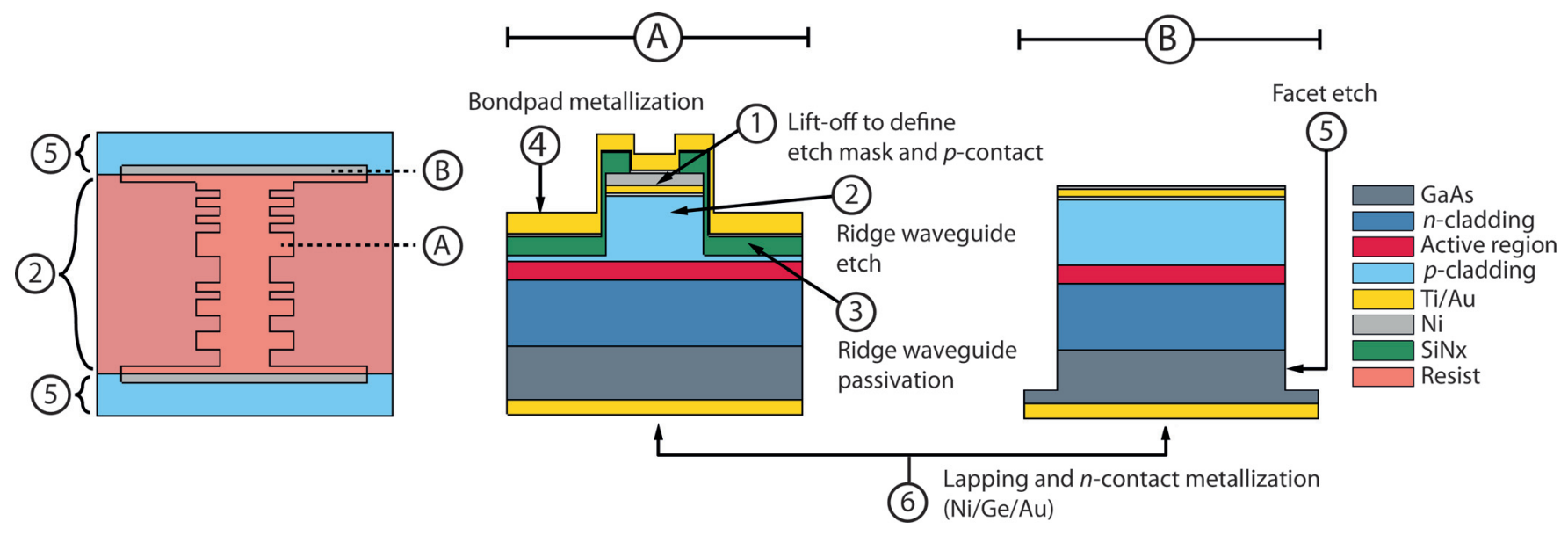

FIG. 4. (Color online) Mask layout (left) and indication of major processing steps that are used in the realization of (a) the perturbed ridge of the waveguide, and (b) the end facet mirrors of the resonator.

different mask materials for facet etching have been investigated in the literature, including conventional ${ }^{9}$ and multiple layer resists, ${ }^{10}$ electron beam resists, ${ }^{11}$ dielectrics,${ }^{12}$ and metallic masks. ${ }^{13,14}$ Among these, metallic masks have proven to be more resistant to erosion, and are therefore preferred for dry etching of deep ( $\gtrsim 2 \mu \mathrm{m})$ features. ${ }^{15}$ Further, by a proper choice of the metal composition of the mask, it can additionally be used for the current injection into the ridge, thus leading to a self alignment of the $p$-contact to the ridge waveguide. For these reasons we chose to use a metallic mask. The metal should be chosen such that the selectivity between the metal and the semiconductor layers is as high as possiblewhich depends on the specific gas chemistry and plasma powers used in the dry etching process-while at the same time it should make a good ohmic contact with the top GaAs layer. In this work, we have used inductively coupled plasma (ICP) dry etching with a $\mathrm{SiCl}_{4}$ - $\mathrm{Ar}$ chemistry suitable for etching of AlGaAs waveguide layers, as explained in more detail in the next section. From measurements on selectivity and contact resistance, we found that a Ti/Au/Ni-mask is highly advantageous to use. The etch selectivity of $\mathrm{Ni}$ to AlGaAs in our etch process is very high, with a typical value of $\sim 80$, while the specific contact resistance was $\sim 10^{-5} \Omega \cdot \mathrm{cm}^{2}$. It is not critical if there is residual $\mathrm{Ni}$ after etching, since this does not significantly influence the contact resistance, which makes it possible to use the Ti/Au/Ni-mask mask also as a $p$-contact.

We define the mask by lithography and lift-off in the very first processing step when the wafer does not yet have a pronounced topography. This makes it possible to obtain a well defined mask with minimally corrugated edges, using thin metal layers. Further, to minimize any end facet surface roughness caused by post etching processing, the ridge waveguide and the facets are dry etched in separate steps with the facets being etched toward the end of the device processing; the ridge waveguide, with its perturbations, is etched earlier in a single step.

\section{FABRICATION OF SPECTRALLY ENGINEERED RESONATORS}

In order to evaluate our fabrication method we designed a number of different SE-FPRs with a varying number of perturbations. The resonators were designed to promote either single mode or two-color operation by lowering the threshold gain for one or two selected longitudinal modes, respectively. All resonators had a length of $500 \mu \mathrm{m}$. The number of perturbations in each resonator design was limited by varying the length of the shortest segment $d$ between $d=270 \mathrm{~nm}$ and $d=2.44 \mu \mathrm{m}$, corresponding to $s=3$ and $s=27$ quarter-wavelengths in the material. When deciding on a desired threshold gain spectrum, used in the design, the amount of threshold gain reduction for the selected modes was chosen according to the number of possible perturbations in each design - using more perturbations enables a larger reduction of threshold gain. For all designs the unperturbed effective index and the index perturbation were set to $n=3.25$ and $\Delta n=-0.0033$, respectively, for all 52 modes within the $\sim 22 \mathrm{~nm}$ bandwidth considered, i.e., dispersion was neglected. All single mode resonators were designed to lower the threshold gain for a mode at $\lambda=1174.6 \mathrm{~nm}$ while the two-color laser resonators were designed to simultaneously lower the threshold gain for two modes at $\lambda=1174.6$ and $\lambda=1169.9 \mathrm{~nm}$. An example of one of these designed two-color resonators, with $d=270 \mathrm{~nm}$, is the one shown in Fig. 1; a summary of some characteristics of all designed resonators is given in Table I.

The epitaxial laser structure used in this study was grown on a GaAs substrate by metal-organic chemical vapor deposition. It consists of an undoped active region including a compressively strained $7 \mathrm{~nm}$ thick InGaAs quantum well, with an In content of $37 \%$, embedded between $40 \mathrm{~nm}$ undoped $\mathrm{Ga}_{0.08} \mathrm{As}_{0.92} \mathrm{P} / \mathrm{GaAs}$ strain compensating barriers. The active region is surrounded by $200 \mathrm{~nm}$ thick graded composition $\mathrm{Al}_{x} \mathrm{Ga}_{1-x}$ As confinement layers $(x=0.2-0.45)$ cladded by $1 \mu \mathrm{m}$ thick $p$ - and $n$-type $\mathrm{Al}_{0.45} \mathrm{Ga}_{0.55}$ As layers doped with $\mathrm{C}$ and $\mathrm{Si}$ at $5 \cdot 10^{17}-1 \cdot 10^{18} \mathrm{~cm}^{-3}$, respectively. A $100 \mathrm{~nm}$ thick $p^{+}$-GaAs layer doped with $\mathrm{C}$ at $3 \cdot 10^{19} \mathrm{~cm}^{-3}$ serves as a contact layer. Fabricated broad-area reference lasers $(w=100 \mu \mathrm{m})$ with cleaved facets emit around 1170 $\mathrm{nm}$ with a threshold current density of $122 \mathrm{~A} / \mathrm{cm}^{2}$ for a 1 $\mathrm{mm}$ long cavity, indicating good material quality.

All SE-FPRs were fabricated on a single chip from this wafer, together with conventional ridge waveguide FPLs 
TABLE I. Summary of characteristics of SE-FPRs designed for single mode and two-color emission.

\begin{tabular}{rccccc}
\hline \hline \multicolumn{2}{c}{ Segment length $^{\mathrm{a}}$} & \multicolumn{2}{c}{ Single mode design } & \multicolumn{2}{c}{ Two-color design } \\
\hline $\mathrm{s}$ & $d(\mathrm{~nm})$ & $N_{p}{ }^{\mathrm{b}}$ & $\Delta \gamma^{\mathrm{c}}$ & $N_{p}{ }^{\mathrm{b}}$ & $\Delta \gamma^{\mathrm{c}}$ \\
27 & 2439 & 55 & 1.0 & 58 & 1.0 \\
11 & 994 & 120 & 1.9 & 112 & 1.9 \\
7 & 633 & 213 & 5.1 & 224 & 3.9 \\
3 & 270 & 500 & 11.8 & $538^{\mathrm{d}}$ & $11.0^{\mathrm{d}}$ \\
\hline \hline
\end{tabular}

${ }^{\text {a }}$ This is the length of the shortest segment in the resonator.

${ }^{\mathrm{b}}$ Number of perturbations.

"Threshold gain reduction $\Delta \gamma$ of the selected "lasing" mode(s), given in percent of the mirror loss of an unperturbed waveguide $\left(\sim 25.5 \mathrm{~cm}^{-1}\right)$.

${ }^{\mathrm{d}}$ This design is shown in Fig. 1.

with a ridge waveguide width of either $w=6 \mu \mathrm{m}$ or $w=3 \mu \mathrm{m}$, and a cavity length of $500 \mu \mathrm{m}$, defined by facet etching as for the SE-FPRs. Three different sets of each SEFPR design were fabricated; the unperturbed ridge width was $w=6 \mu \mathrm{m}$ for all sets while the perturbed waveguide width was either $w=3.0 \mu \mathrm{m}, w=3.4 \mu \mathrm{m}$ or $w=4.1 \mu \mathrm{m}$ in a given set, corresponding to ridge width modulations of $\Delta w=3.0 \mu \mathrm{m}, \Delta w=2.6 \mu \mathrm{m}$, and $\Delta w=1.9 \mu \mathrm{m}$, respectively. Figure 4 indicates the most important steps in the fabrication process. Processing is initiated by defining the $p$ contact also used as the masking material for etching the perturbed ridge waveguide and the facets. Either direct-write electron-beam lithography (EBL) or conventional lithography can be used, depending on the smallest feature size of the laterally corrugated waveguides (i.e., $d$, the length of an isolated perturbed or unperturbed cavity segment). For feature sizes larger than $\sim 1 \mu \mathrm{m}$ it is possible to use a standard image reversal process with photoresist for pattern definition and electron-beam evaporation of the metal films. However, in this work we have used direct-write EBL since the feature size is well below $1 \mu \mathrm{m}$ for several of the SE-FPRs. In this process, the chip is first spin-coated with a bi-layer resist stack of MMA-MAA copolymer (MMA(8.5)MAA) at the bottom and high resolution ZEP520 A at the top. The etch mask pattern, which includes all SE-FPRs and FPLs, is subsequently transferred to the sample via an electron-beam exposure in a JEOL JBX-9300FS system (the patterns were proximity effect compensated by dose correction) followed by development using n-amyl acetate and MIKB:IPA (1:1) for development of the ZEP520 A and copolymer resists, respectively. The sample is rinsed in IPA and blow-dried with $\mathrm{N}_{2}$ immediately after development. Using two different resists that require two different developers makes it easier to control the resist undercut profile needed for a successful lift-off process. Next, the combined $p$-contact and etch mask is finalized by depositing $\mathrm{Ti} / \mathrm{Au} / \mathrm{Ni}$ with electron-beam evaporation followed by a standard lift-off step.

The thicknesses of the Ti/Au layers, incorporated to create a $p$-contact with a low resistance, are not crucial. However, since the Ni-layer serves as an etch mask its thickness must be chosen such that it can withstand etching of both the ridge waveguide and the end facets. We use ridge waveguide and end facet etch depths of 1.4 and $5 \mu \mathrm{m}$, respectively, which together with a typical etch selectivity of $80: 1$ between $\mathrm{Ni}$ and $\mathrm{AlGaAs}$ in our dry etch process implies that at least an $\sim 80 \mathrm{~nm}$ thick $\mathrm{Ni}$ layer is required. To have some margin we have used a Ni thickness of $90 \mathrm{~nm}$ together with $\mathrm{Ti}$ adhesion and Au layer thicknesses of 10 and $40 \mathrm{~nm}$, respectively, thus yielding a total $p$-contact thickness of 140 $\mathrm{nm}$. In order to avoid etching the facets during the ridge waveguide etching a pattern of positive AZ1512 resist is applied, with the edge of the resist aligned to the center of the $2 \mu \mathrm{m}$ long T-bar segments at both ends of the waveguide. The ridge waveguides are etched in an ICP etcher under low pressure $(<1 \mathrm{~Pa})$ using $\mathrm{SiCl}_{4}$ and $\mathrm{Ar}$ flows of 10 and 20 sccm, respectively, together with low inductive source and substrate bias powers of 55 and $50 \mathrm{~W}$ in order to reduce surface damage of the etched areas. This is important since rough side walls may cause significant light scattering of the optical mode; this should be particularly important for the SE-FPR with its narrow sections along the ridge waveguide. The resulting etch rate was $120 \mathrm{~nm} / \mathrm{min}$ for the AlGaAs layers and slightly higher for the GaAs layers. The ridges are etched to a depth of $1.4 \mu \mathrm{m}$ which leaves $0.1 \mu \mathrm{m}$ of the upper cladding layer above the active region. The etching system allows for precise depth control through a laser interferometer end point detection system, which is important since the magnitude of the obtained index perturbation $\Delta n$ depends strongly on the etch depth, as shown in Fig. 3. The sample is rinsed for 60 seconds in de-ionized water immediately after removal from the ICP chamber. This is necessary to prevent corrosion, caused by chlorinated etch product residues, of the $\sim 70 \mathrm{~nm}$ thick $\mathrm{Ni}$ film remaining on top of the ridge waveguide after etching. The so obtained ridge waveguide sidewalls are vertical and smooth, as illustrated in the tilted view scanning electron micrograph in Fig. 4. To electrically isolate the ridge waveguide, a $160 \mathrm{~nm}$ thick layer of $\mathrm{SiN}_{x}$ is subsequently deposited on the entire chip surface with plasma-enhanced chemical vapor deposition, after which 1.0 $\mu \mathrm{m}$ wide openings, centered on top of the ridge waveguides, are defined in AZ1512 resist and etched using ICP etching with $\mathrm{NF}_{3}(20 \mathrm{sccm})$ using 30 and $300 \mathrm{~W}$ source and substrate bias powers, respectively. Next, $100 \times 490 \mu \mathrm{m}^{2} \mathrm{Ti}(15 \mathrm{~nm}) /$

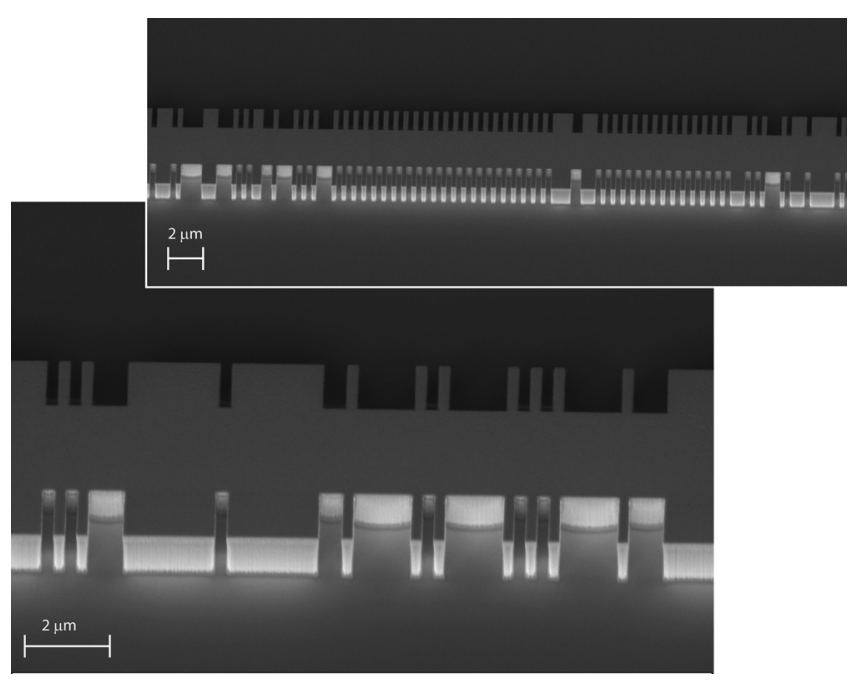

FIG. 5. SEM image of the perturbed ridge of a fabricated SE-FPR, showing the high definition and smoothness of the etched side walls. 
$\mathrm{Au}(250 \mathrm{~nm})$ bond pads are patterned and sputtered on top of the ridge waveguides followed by patterning with AZ1512 resist for protection of the ridge waveguides and bond pads during facet etching, with the protective mask being positioned between the centers of the T-bar segments of the ridge waveguide, as shown in Fig. 4. The facets are then ICP etched using the same conditions as used previously for the ridge waveguide etching. The etch depth is about $5 \mu \mathrm{m}$ which corresponds to a complete removal of the epitaxial structure and an additional $\sim 1.8 \mu \mathrm{m}$ etch into the GaAs substrate. The obtained facet surfaces are smooth and vertical as shown in Fig. 6. After the facets have been etched the substrate is thinned to approximately $150 \mu \mathrm{m}$ (during which the sample surface is protected with resist) before a $\mathrm{Ni} / \mathrm{Ge} / \mathrm{Au} n$ contact layer is evaporated on the entire backside of the chip, followed by a rapid thermal annealing treatment of the $n$ contact at $430{ }^{\circ} \mathrm{C}$ for $30 \mathrm{~s}$. Finally, the sample is cleaved at positions approximately $100 \mu \mathrm{m}$ in front of the etched facets in order to have access to the facet surfaces for light-current (L-I) measurements. A tilted view SEM image of a fabricated device is shown in Fig. 7.

\section{MEASUREMENTS AND ANALYSIS}

The electrical and optical characteristics of the fabricated lasers were measured under continuous-wave conditions at a heat sink temperature of $25^{\circ} \mathrm{C}$. The light output from one of the facets was detected with a large-area InGaAs photodetector while a butt coupled standard multimode fiber was used to simultaneously collect the laser light emitted from the other facet. The fiber was positioned at an angle in front of the facet to minimize reflections from the fiber back into the laser resonator. A fiber-coupled optical spectrum analyzer with a resolution of $0.01 \mathrm{~nm}$ was used to record the emission spectra.

The quality of an etched facet is reflected in the L-I characteristics of the laser since a reduced reflectivity caused by scattering and/or nonvertical facets would result in a higher threshold current $\left(I_{\mathrm{th}}\right)$ as well as a lower slope efficiency $(\Delta P / \Delta I)$. For this investigation we used only the

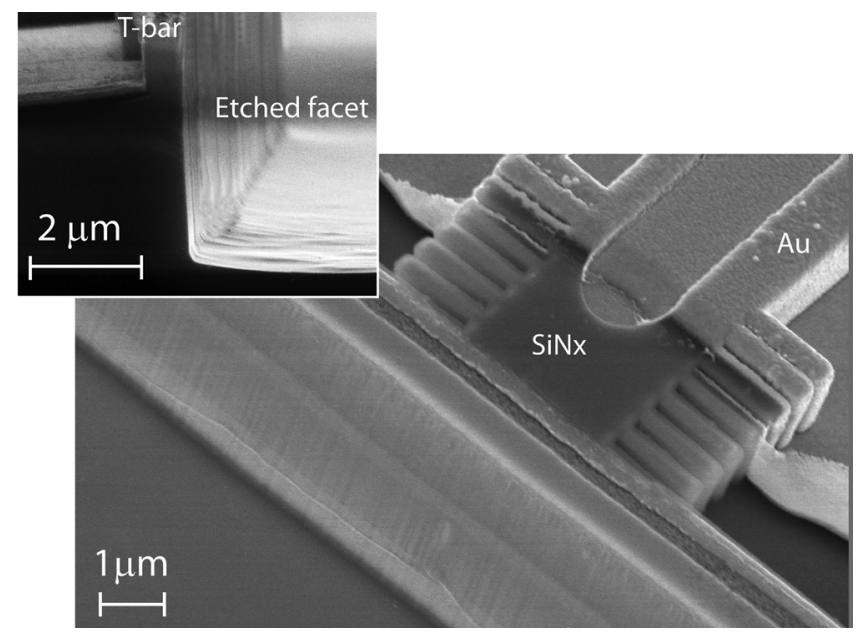

FIG. 6. One of the etched end facet mirrors of the resonator. The cross section of an etched facet is seen in the inset, showing the smooth and vertical mirror surface.

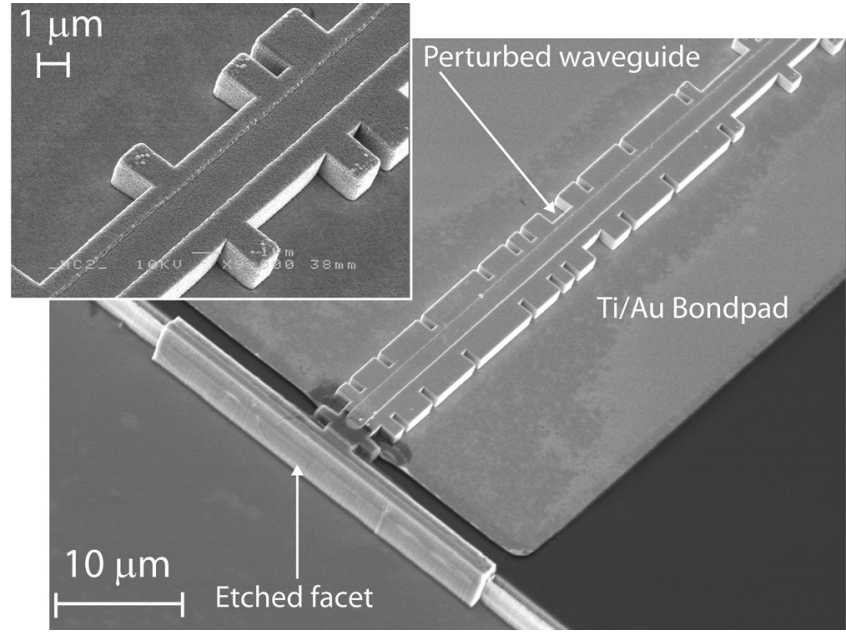

FIG. 7. SEM image of a fabricated device illustrating one etched facet and the perturbed waveguide.

FPLs. The L-I characteristics for $6 \mu \mathrm{m}$ wide FPLs, all fabricated from the same wafer, with both facets either cleaved or etched are shown in Fig. 8. The length of the lasers with etched facets was lithographically defined to be $500 \mu \mathrm{m}$ while the length of the lasers with cleaved facets was measured with an optical high magnification microscope to be somewhat larger, $\sim 580 \mu \mathrm{m}$. For the lasers with cleaved facets, typical $I_{\text {th }}$ and $\Delta P / \Delta I$-values of $10 \mathrm{~mA}$ and $0.46 \mathrm{WA}^{-1}$, respectively, were obtained. The corresponding values for the lasers with etched facets were $11.5 \mathrm{~mA}$ and $0.46 \mathrm{WA}^{-1}$, respectively. Although there is a small difference in resonator length, the similarity of these values suggests that the etched facets are of high quality; a more detailed quantitative analysis is difficult because of the uncertainties in some parameter values but indicates that if the perfectly cleaved facet has a (Fresnel) reflectivity of $30 \%$, the etched facets would have a reflectivity of $22-25 \%$.

Next, the lasers with SE-FPRs were characterized. Measurements of the threshold current as a function of the number of introduced perturbations $\left(N_{p}\right)$ were performed to

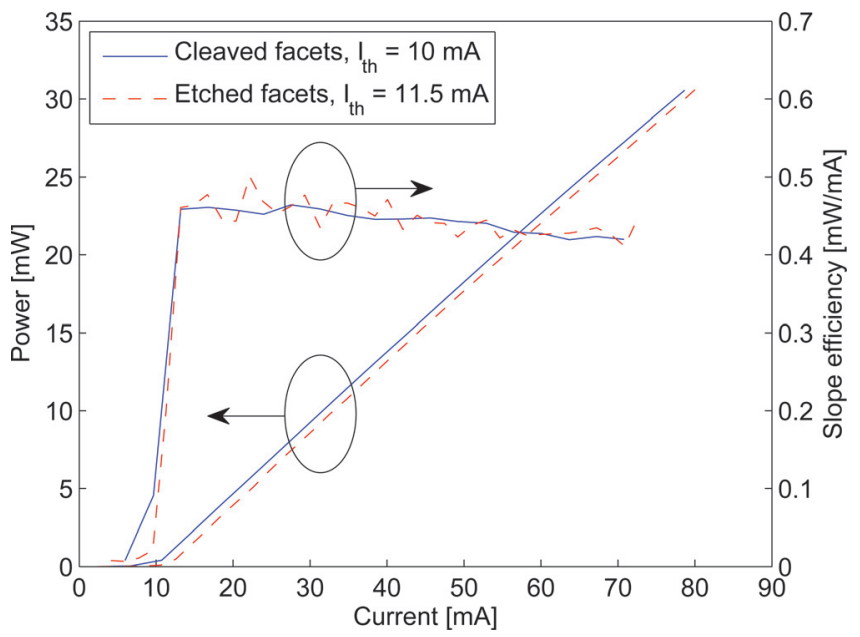

FIG. 8. (Color online) Measurements on FPLs: output power and slope efficiency as functions of the injection current for lasers with cleaved and etched end facet mirrors. 


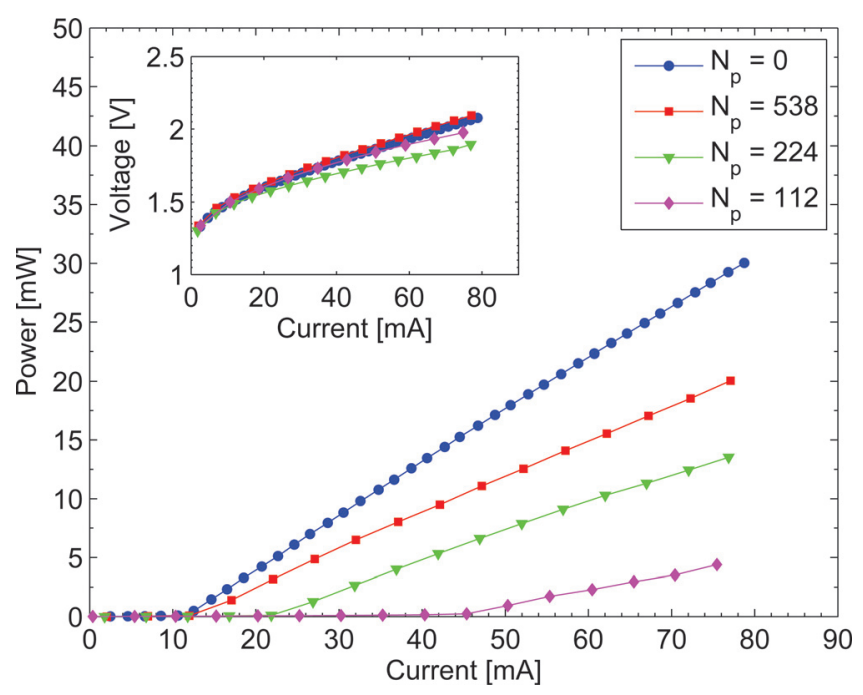

FIG. 9. (Color online) Measurements on lasers with SE-FPRs: output power and applied voltage (inset) as functions of the injection current for lasers with different number of perturbations, $N_{p}$. The ridge width modulation $\Delta w=3 \mu \mathrm{m}$.

examine the additional propagation loss caused by the perturbations. Figure 9 displays typical L-I characteristics for a range of lasers with a ridge width modulation $\Delta w=3 \mu \mathrm{m}$. As can be seen, the perhaps slightly surprising result is that the threshold current decreases with the number of perturbed cavity segments. To coarsely quantify the additional propagation loss we used a simple logarithmic dependence of material gain on current density approximately valid for quantum well materials. ${ }^{7}$ Then the additional loss can be written as

$$
\Delta \alpha=\alpha_{\mathrm{SE}-\mathrm{FPR}}-\alpha_{\mathrm{FPL}}=\Gamma g_{0} \ln \left(\frac{I_{\mathrm{th}, \mathrm{SE}-\mathrm{FPR}}}{I_{\mathrm{th}, \mathrm{FPL}}}\right),
$$

where $\alpha$ is the propagation loss (internal optical loss) of the optical mode, $\Gamma$ its confinement factor, and $g_{0}$ is a material gain coefficient. The uncertainty $\pm \sigma$ in the determination of $\Delta \alpha$ is estimated as a root mean square

$$
\sigma=\sqrt{\left(\frac{\partial \Delta \alpha}{\partial x} \sigma_{x}\right)^{2}+\left(\frac{\partial \Delta \alpha}{\partial y} \sigma_{y}\right)^{2}+\left(\frac{\partial \Delta \alpha}{\partial z} \sigma_{z}\right)^{2}}
$$

where $x, y$, and $z$ are introduced as short notations for $\Gamma g_{0}, I_{\text {th }, \text { SE-FPR }}$, and $I_{\text {th }}$, FPL, respectively, the derivatives are calculated from Eq. 1 , and $\sigma_{x}, \sigma_{y}$, and $\sigma_{z}$ denote the estimated uncertainty in the values for these parameters. The additional loss is plotted in Fig. 10 as a function of the number of perturbations, $N_{p}$, and ridge width modulation $\Delta w$. For the calculations a value for $\Gamma g_{0}=35 \pm 5 \mathrm{~cm}^{-1}$ was used, which was obtained from cavity-length dependent L-I measurements on broad-area lasers. From the figure, we observe a decrease in additional propagation loss from $53 \pm 11.1 \mathrm{~cm}^{-1}$ to $10.0 \pm 2.1 \mathrm{~cm}^{-1}$ when $N_{p}$ is increased from $\sim 100$ to $\sim 500$, for $\Delta w=3.0 \mu \mathrm{m}$. From Hakki-Paoli measurements of the net modal gain ${ }^{16}$ the propagation loss of an FPL was determined to be $\alpha_{\mathrm{FPL}} \sim 20 \mathrm{~cm}^{-1}$ yielding a total resonator loss, consisting of the propagation loss, mirror loss, and additional loss caused by the perturbations, of $\sim 60 \mathrm{~cm}^{-1}$ for an

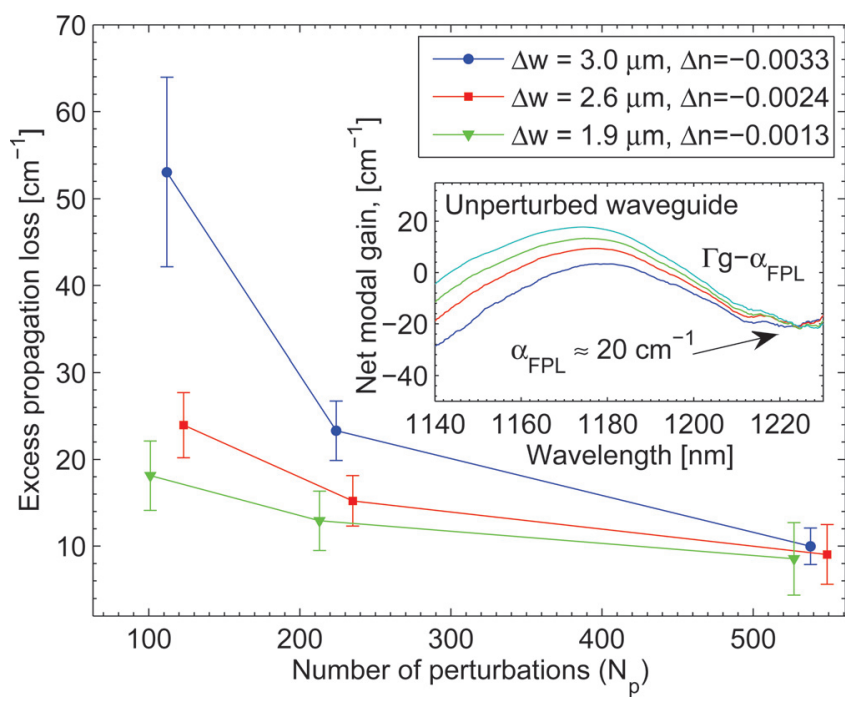

FIG. 10. (Color online) Estimations of the excess propagation loss, i.e., the additional loss caused by the perturbations, compared to an unperturbed resonator. The estimation is based on a threshold current analysis according to Eq. (1) and the error bars are calculated using Eq. (2), where the uncertainties are estimated from measurements on several, nominally identical, lasers. Note that the obtained values for $\Delta w=2.6 \mu \mathrm{m}$ and $\Delta w=1.9 \mu \mathrm{m}$ have been shifted slightly along the $N_{p}$-axis for clarity. The inset shows results from Hakki-Paoli measurements of the net modal gain for an FPL, for four different bias currents, from which the propagation loss value, $\alpha_{\mathrm{FPL}}$, can be estimated.

SE-FPR with $N_{p} \approx 500$, where $R=25 \%$ was used for estimating the mirror loss. The additional loss thus constitutes $\sim 15 \%$ of the total loss in this case. Further, the measured series resistance was as low as $\sim 5-7$ ohms for all devices, demonstrating that the $\mathrm{Ti} / \mathrm{Au} / \mathrm{Ni} / \mathrm{Ti} / \mathrm{Au} p$-contact achieved a low contact resistance.

One reason for the higher loss for an SE-FPR with a low number of perturbations might be that the narrow segments are so few that the optical field propagates long distances in unperturbed, broad, portions of the waveguide, and thus that the mode size of the optical field adjusts to the broad waveguide. Once the field reaches a narrow portion of the ridge, there will be a large mode mismatch and a relatively large field at the edge of the narrow ridge, causing additional optical loss through scattering and/or absorption in the sputtered $\mathrm{Ti} / \mathrm{Au}$ bond pad layer. On the other hand, if the perturbations are dense, as for an SE-FPR with large $N_{p}$, the mode should adjust to the average width of the waveguide, making the modal width constant throughout the length of the resonator ${ }^{6}$ and thereby avoiding a large mismatch between the propagating mode and any part of the waveguide. This is further supported by a significant decrease in excess propagation loss for $N_{p} \lesssim 200$ when the ridge width modulation is reduced, as seen in Fig. 9. For resonators with $N_{p} \sim 100$, the excess loss is for example decreased from $\Delta \alpha=53 \pm 11.1 \mathrm{~cm}^{-1}$ to $\Delta \alpha=23.9 \pm 3.8 \mathrm{~cm}^{-1}$, when $\Delta w$ is reduced from $3.0 \mu \mathrm{m}$ to $2.6 \mu \mathrm{m}$.

Although the fabricated SE-FPRs cannot be expected to exactly produce the desired laser spectra (since there are uncertainties in some material parameters), we should be able to establish that the perturbations at least strongly influence the spectral behavior. To examine this, emission spectra 
were recorded as a function of bias current for both FPLs and lasers with SE-FPRs. The lower part of Fig. 11 shows the evolution of peak emission wavelength(s) with current for an FPL and for lasers with SE-FPRs designed for single mode and two-mode emission, respectively. Also shown in Fig. 11 are examples of recorded emission spectra. For the displayed single mode SE-FPR, at a bias current above $\sim 1.1 I_{\text {th }}$ a single longitudinal mode at $1174.1 \mathrm{~nm}$ was preferentially enhanced, yielding a side-mode-suppression-ratio (SMSR) of $>30 \mathrm{~dB}$. The device oscillated at this mode for bias currents up to $\sim 4.5 I_{\text {th }}$ with maintained SMSR. As can be seen, the measured peak wavelength shift with bias current was $0.03 \mathrm{~nm} / \mathrm{mA}$. This value is roughly 3 times lower than the shift of the gain peak with current, the latter which is given by the rate of the shift in peak emission wavelength of the FPL, as shown. This indicates that in the SE-FPR the lasing mode was preferentially selected by mode-selective optical feedback and not by the gain peak, whereas in the FPL no such strongly mode-selecting mechanism was evident. A similar single mode emission behavior was observed for all devices with spectrally engineered single-mode resonators, in the entire range of different values for $N_{p}$, although the selected mode differed within $\sim 5$ longitudinal modes between devices. As is seen in the figure, also the SE-FPRs designed for two-mode emission exhibit the slow dependence of wavelength on current which indicates that the
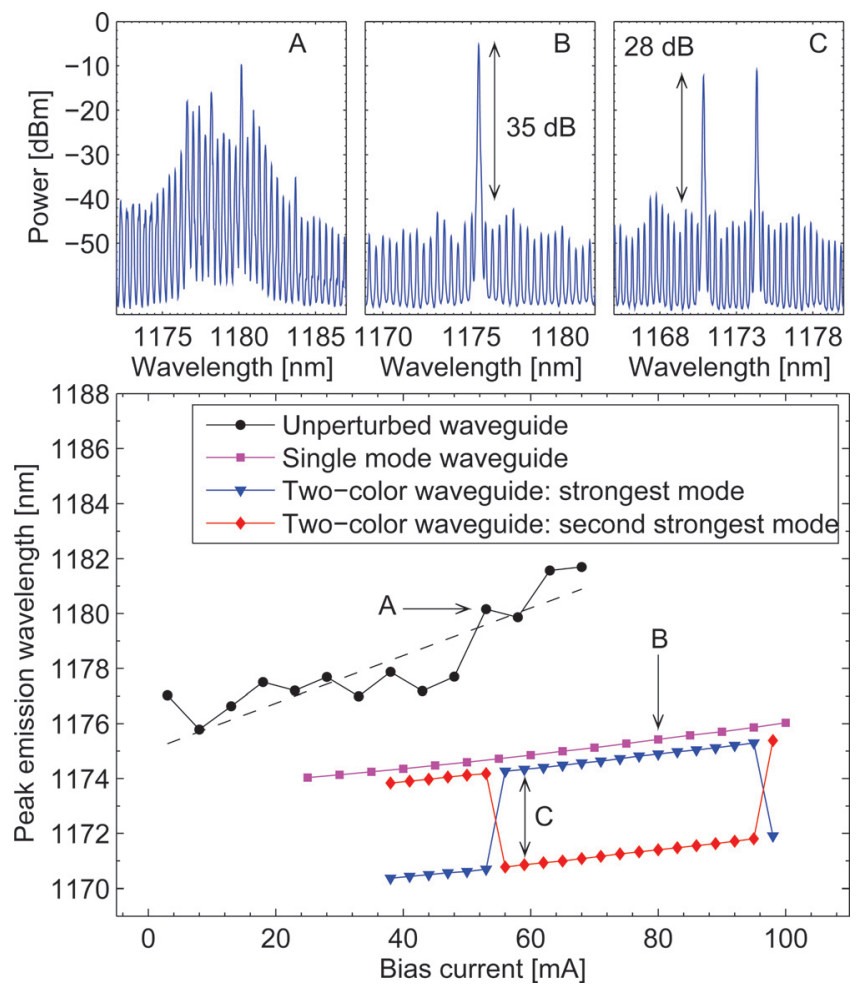

FIG. 11. (Color online) Results from spectral measurements on FPLs and lasers with SE-FPRs: Bottom: peak wavelength (at which the intensity is maximum) as a function of injected current, for an FPL (a) and for lasers with SE-FPRs designed for single-mode (b), and two-mode operation (c), respectively. The dashed line illustrates the trend in increasing peak wavelength with bias current for the FPL (which is $\sim 3$ times larger than the increase of $0.03 \mathrm{~nm} / \mathrm{mA}$ obtained for SE-FPRs). Top: examples of emission spectra, recorded at bias currents as indicated in the lower plot. emission wavelength is governed primarily by modal preference rather than gain. However, only for a small bias current range were the two modes approximately equal in power; at the optimal bias current where the two modes have identical power an SMSR of $\sim 30 \mathrm{~dB}$ was obtained. Also, some twomode resonators had single-mode emission throughout the entire range of bias currents, which likely implies that the two-mode designs are more sensitive to imperfections in the realization of the etched structures and uncertainties in the material parameters. In spite of this sensitivity, any specific laser remained stable in its spectral characteristics with respect to time and operating conditions: measurements after several weeks, and after having used such high bias currents that the lasers switched to lasing at the first excited state in the quantum well, produced the same spectra as the initial measurements on the same device.

\section{CONCLUSIONS}

In this work, we have developed methods for the highly precise geometrical definition of spectrally engineered Fabry-Perot resonators with low optical loss. Self-alignment of the perturbations relative the resonator end facets as well as self-alignment of the $p$-contact to the ridge waveguide was critical in this realization. In addition, the mask layout as well as the use of separate dry etching steps for defining the waveguide and facets resulted in a process well suited for obtaining etched facets with a low surface roughness. Also, a careful choice of etch mask materials that serve several purposes simplified the processing. Measurements on fabricated lasers indicate that the etched structures are of high surface quality and high verticality, the latter which is particularly important for the deeply etched end facet mirrors. Moreover, the measured variation of the emission wavelength with bias current shows that the spectral behavior is governed by the partial reflections from the resonator perturbations, although uncertainties in material gain and refractive index cause deviations from the desired spectra.

\section{ACKNOWLEDGMENTS}

This work was supported by the Swedish Research Council (Vetenskapsrådet), Project No. 621-2010-3537.

${ }^{1}$ S. O’Brien, A. Amann, R. Fehse, S. Osborne, E. P. O'Reilly, and J. M. Rondinelli, J. Opt. Soc. Am. B: Opt. Phys. 23, 1046 (2006).

${ }^{2}$ S. Osborne, S. O'Brien, K. Buckley, R. Fehse, A. Amann, J. Patchell, B. Kelly, D. R. Jones, J. O'Gorman, and E. P. O'Reilly, IEEE J. Sel. Topics Quantum Electron. 13, 1157 (2007).

${ }^{3}$ S. O'Brien, S. Osborne, D. Bitauld, and A. Amann, Photonics Nanostruct. Fundam. 8, 218 (2010).

${ }^{4}$ G. Adolfsson, J. Bengtsson, and A. Larsson, J. Opt. Soc. Am. B: Opt. Phys. 27, 118 (2010).

${ }^{5}$ D. A. Francis, C. J. Chang-Hasnain, and K. Eason, Appl. Phys. Lett. 68, 1598 (1996).

${ }^{6}$ A. Laakso, M. Dumitrescu, J. Viheriälä, J. Karinen, M. Suominen, and M. Pessa, Opt. Quantum Electron. 40, 907 (2008).

${ }^{7}$ L. A. Coldren and S. W. Corzine, Diode Lasers and Photonic Integrated Circuits, edited by L. A. Coldren (John Wiley \& Sons, Inc., 1995).

${ }^{8}$ M. A. Afromowitz, Solid State Commun. 15, 59 (1974).

${ }^{9}$ S. C. Horst, S. Agarwala, O. King, J. L. Fitz, and S. D. Smith, Appl. Phys. Lett. 71, 1444 (1997).

${ }^{10}$ G. L. Bona, P. Buchmann, R. Clauberg, H. Jaeckel, P. Vettiger, O. Voegeli, and D. J. Webb, IEEE Photon. Technol. Lett. 3, 412 (1991). 
${ }^{11}$ M. Hagberg, B. Jonsson, and A. Larsson, J. Vac. Sci. Technol., B 10, 2243 (1992).

${ }^{12}$ S. A. Biellak, C. G. Fanning, Y. Sun, S. S. Wong, and A. E. Siegman, IEEE J. Quantum Electron.33, 219 (1997).

${ }^{13}$ G. M. Smith, D. V. Forbes, J. J. Coleman, and J. T. Verdeyen, IEEE Photon. Technol. Lett. 5, 873 (1993).
${ }^{14}$ I. E. Johnson and C. L. Tang, IEEE Photon. Technol. Lett. 4, 24 (1992).

${ }^{15}$ J. R. Lothian, F. Ren, and S. J. Pearton, Semicond. Sci. Technol. 7, 1199 (1992).

${ }^{16}$ B. W. Hakki and T. L. Paoli, J. Appl. Phys. 46, 1299 (1975). 